\title{
Very Steep Optical Thresholding Characteristic Using a DFB Laser Diode and an SOA in an Optical Feedback Scheme
}

\author{
W. D’Oosterlinck, Student Member, IEEE, G. Morthier, Senior Member, IEEE, M. K. Smit, Fellow, IEEE, and \\ R. Baets, Senior Member, IEEE
}

\begin{abstract}
A new optical decision or thresholding circuit, composed of a semiconductor optical amplifier (SOA) and a distributed feedback (DFB) laser diode in an optical feedback scheme is proposed. This new circuit has been studied both by simulations and experiments. Simulations show a very steep optical decision characteristic that rises more then $5 \mathrm{~dB}$ over an input power range of less then $0.5 \mathrm{~dB}$. It is also demonstrated that the decision point can easily be shifted by tuning the drive currents of both the laser diode or the SOA or the feedback ratio between them. The principle has been experimentally verified as well, using a discrete SOA and DFB laser diode. We showed a good agreement with the simulation results. Again a very steep optical decision characteristic, combined with the possibility to adjust the decision point was obtained.
\end{abstract}

Index Terms-Distributed feedback (DFB) laser diode, optical decision characteristic, optical feedback, semiconductor optical amplifier (SOA).

\section{INTRODUCTION}

$\mathbf{O}$ PTICAL thresholding circuits will be key elements in future all-optical networks. They can not only be used in optical regenerators but can also find applications in other fields such as optical signal monitoring [1], optical packet header processing [2], etc. Different approaches to come to optical thresholding functions have been suggested before. Although most of them are based on the use of semiconductor optical amplifiers (SOAs) in interferometric configurations, results have also been reported using bistable lasers [3] and saturable absorbers [4]. Different schemes using interferometric schemes incorporating SOAs have been developed, such as a semiconductor laser amplifier loop optical mirror or other types of fiber based nonlinear interferometers [5] and integrated Mach-Zehnder or Michelson interferometers. The fiber-based nonlinear interferometers can be very fast but are also quite complicated to fabricate. The Mach-Zehnder interferometer-based devices have the possibility to be integrated

\footnotetext{
Manuscript received July 15, 2004; revised October 20, 2004. This work was supported in part by the European Commission through the IST-STOLAS Project, in part by the IST-Nefertiti Network, and in part by the Belgian DWTC through the IAP5/18 Project. The work of W. D'Oosterlinck was supported by the Flemish Institute for the Industrial Advancement of Scientific and Technological Research (IWT) under a specialization grant.

W. D'Oosterlinck, G. Morthier, and R. Baets are with the Photonics Research Group of the Department of Information Technology (INTEC), Ghent University-IMEC, Gent 9000, Belgium (e-mail: wouter.doosterlinck@intec.ugent.be).

M. K. Smit is with the Department of Electrical Engineering, COBRA Research Institute, Eindhoven University of Technology, Eindhoven 5600 MB, The Netherlands.

Digital Object Identifier 10.1109/LPT.2004.842322
}

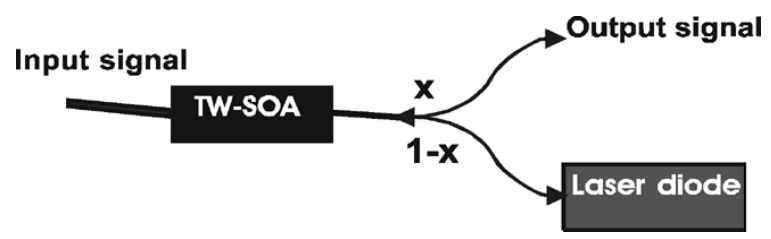

Fig. 1. Schematic representation of the newly proposed optical decision circuit.

on a single chip but often suffer from other disadvantages, e.g., it is not easy to obtain both high-speed operation [6], [7] and steep decision characteristics [8], [9] with the same component. Furthermore, the need for always higher speed makes the devices more and more complex resulting in higher costs. The major setback for the use of interferometric devices is, however, the sensitivity of the nonlinear effects to external factors like the temperature and pressure.

Here we present a new optical decision circuit, which does not rely on interference but simply consists of a bidirectional optical feedback scheme between an SOA and a distributed feedback (DFB) laser diode. The first simulations and experiments indicate that a very steep optical decision characteristic can be obtained using this scheme. The scheme is fairly simple and with proper design of both the SOA and the DFB laser diode we believe high-speed operation can be achieved in the future.

\section{PRINCIPLE OF OPERATION}

The newly proposed optical decision circuit is shown schematically in Fig. 1. The circuit consists of a travelling wave SOA (TW-SOA) connected to a laser diode by means of a splitter-combiner. A fraction of the output power of the SOA is injected into the laser diode. The same fraction of the laser power is injected back into the SOA. The fraction of the output power of the SOA that is not coupled to the laser will give us the output power of the circuit. The laser should be an antireflection (AR)-coated DFB laser with Bragg wavelength sufficiently distant from the signal wavelength in order to avoid reflection at the laser diode.

When a low input signal is injected into the SOA, the power coupled into the laser is small, resulting in a high output power of the laser diode. This means that a high (laser) power is injected into the right-hand side facet of the SOA which gives rise to an asymmetric spatial hole burning, as can be seen in Fig. 2(a). This asymmetrical carrier distribution, combined with the high laser power that saturates the SOA causes a small gain 


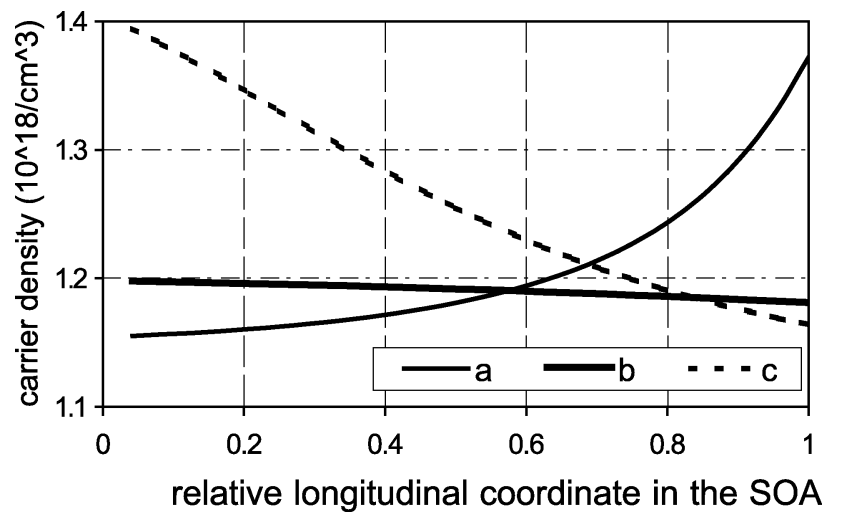

Fig. 2. Schematic representation of the carrier distribution along the longitudinal coordinate of the SOA for different input powers. (a) Low input power. (b) Medium input power. (c) High input power.

for the input signal. At higher input powers, the output power of the SOA rises, resulting in lower output powers of the laser which in its turn results in a change in the spatial hole burning of the SOA to a more symmetric distribution, as can be seen in Fig. 2(b), and a higher gain for the input signal. Obviously, the higher gain results in an even higher injection into the DFB and an even lower injection into the right-hand side facet of the SOA. At a certain input power, which is, however, difficult to predict, this multiplicative effect between the decreasing laser power and the increasing gain for the input signal becomes so strong that a step occurs in the output power of the SOA. At that moment, the spatial hole burning in the SOA is predominantly determined by the input signal as opposed to the laser signal at lower input powers [Fig. 2(c)]. The step in the output power corresponds with a switching of the spatial hole burning from the situation given in Fig. 2(a) to the situation in Fig. 2(c).

Since we can see to it that the SOA remains constantly in saturation, either by a high input power or a high laser power, it is possible to obtain high-speed operation provided we can avoid the laser to turn completely OFF at high input powers (from the SOA). This can be achieved by tuning the currents of both SOA and laser in such a way that the output saturation power of the SOA, after going through the splitter-combiner, causes the laser to operate just above threshold thus reducing the relaxation oscillations of the laser at switch ON.

\section{Simulation RESUlts}

First, some simulations on the new optical decision circuit were performed using commercially available software. ${ }^{1}$ The setup used in the simulation is the same as shown in Fig. 1. The SOA is a bulk SOA with length $500 \mu \mathrm{m}$. The laser is an AR-coated $\lambda / 4$-shifted DFB laser with length $500 \mu \mathrm{m}$ and $\kappa L_{d}=3$. The Bragg wavelength of the laser is $1552 \mathrm{~nm}$. To reduce reflections (due to coupling into the Bragg reflector) of the signal light in the laser back to the SOA the signal wavelength is chosen at $1542 \mathrm{~nm}$.

In Fig. 3, the simulated static decision characteristics are given for different drive currents to both SOA and laser diode. These decision characteristics are very steep, nearly digital.

${ }^{1}$ VPItransmissionmaker and VPIcomponentmaker, VPIsystems. Inc.

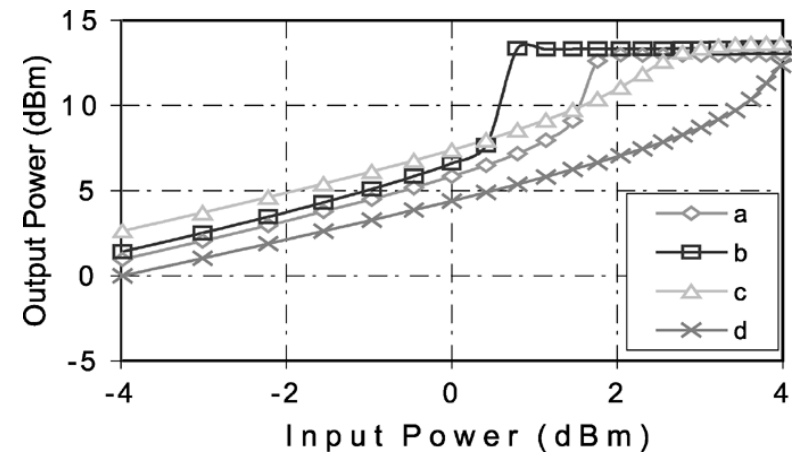

Fig. 3. Calculated static decision characteristics for different coupling factors and drive currents of both SOA and laser diode. (a) $x=0.5, I_{\text {laser }}=120 \mathrm{~mA}$, and $I_{\mathrm{SOA}}=120 \mathrm{~mA}$. (b) $x=0.5, I_{\text {laser }}=120 \mathrm{~mA}$, and $I_{\mathrm{SOA}}=130 \mathrm{~mA}$. (c) $x=0.6, I_{\text {laser }}=120 \mathrm{~mA}$, and $I_{\mathrm{SOA}}=120 \mathrm{~mA}$. (d) $x=0.5, I_{\text {laser }}=$ $140 \mathrm{~mA}$, and $I_{\mathrm{SOA}}=120 \mathrm{~mA}$.

The output power rises more then $5 \mathrm{~dB}$ over an input power range of less than $0.5 \mathrm{~dB}$ [Fig. 3, curve (a) and (b)]. The output power of the SOA increases slowly for low input powers, which could make it difficult to obtain good noise suppression at the zero-level. This slowly rising slope is an intrinsic quality of the circuit as for rising (low) input powers the spatial hole burning becomes less determined by the laser and more by the incoming signal light. At a certain point, called the decision point, the slope becomes steeper. From this point on, the spatial hole burning in the SOA is predominantly determined by the input power as the output power of the SOA becomes high enough to start pushing down the laser power. Eventually when the output power of the SOA equals the saturation output power of the SOA, a flat one-level is reached.

We also show the possibility to shift the decision point by adjusting the coupling factor or the currents of the laser diode or the SOA. When increasing the coupling factor (and decreasing the feedback between the SOA and the laser diode) or the current of the laser diode, the decision point shifts to higher input powers and the jump in output power becomes less steep as compared to Fig. 3(a). This is caused by the fact that in these cases, an increase in the SOA output power will have less influence on the output power of the laser diode. As a result, the nonlinear interplay of the laser power and SOA output power becomes smaller which explains the behavior mentioned above. When the current of the SOA is increased, the opposite effect occurs resulting in a shift of the decision point to lower input powers and a steeper step in the output power.

\section{EXPERIMENTAL RESULTS}

Experiments were performed on an implementation of the circuit with discrete components. The coupler-combiner is a fixed 60/40 coupler $(x=0.4)$. The DFB laser diode used is a fiber-pigtailed DFB laser without isolator and with AR-coated facets.

In Fig. 4, we show an experimentally obtained optical decision characteristic. The signal wavelength is $1550 \mathrm{~nm}$ and the laser wavelength is $1560 \mathrm{~nm}$. The drive currents for SOA and laser are 150 and $22 \mathrm{~mA}$, respectively. The low drive current for the laser is needed because the bad coupling between fiber and the laser forces us to operate the laser close above threshold 


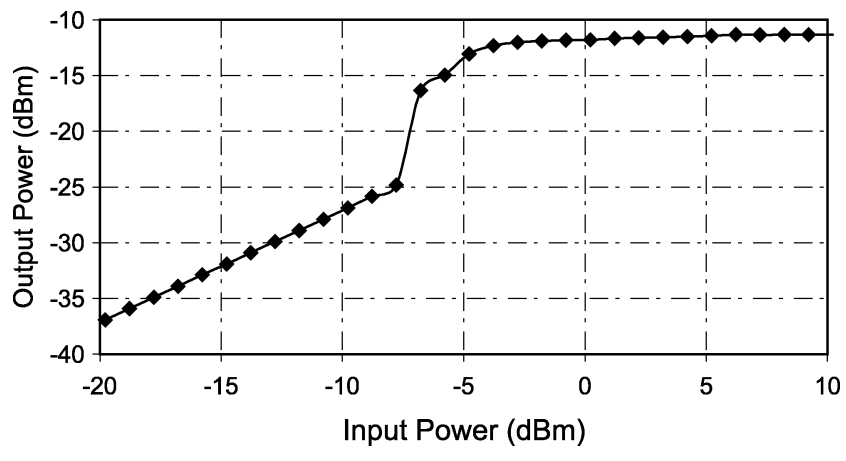

Fig. 4. Experimental static decision characteristic with $I_{\mathrm{SOA}}=150 \mathrm{~mA}$ and $I_{\text {laser }}=22 \mathrm{~mA}$.

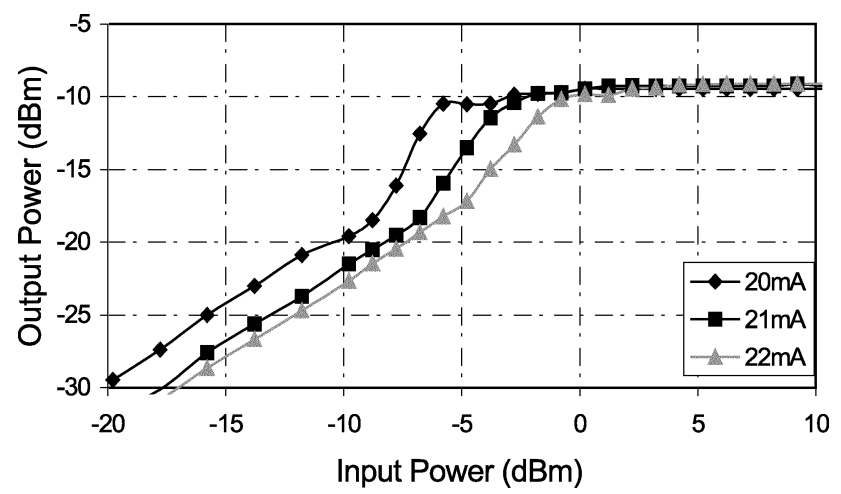

Fig. 5. Demonstration of the possibility to shift the decision point by tuning the drive current of the laser diode.

to obtain the change in laser power that is needed for the multiplicative effect in the circuit. For this reason, we also amplified the laser power before injecting it into the SOA, using an erbium-doped fiber amplifier and circulator between the coupler-combiner and the laser diode.

The output power exhibits a very steep step of about $10 \mathrm{~dB}$ over an input power range of $1 \mathrm{~dB}$. It can be seen that the experimentally obtained characteristic corresponds to the characteristics obtained in the simulations. We observe the same slow rising slope at low input powers and the flat one-level for higher input powers.

To move the decision point, only the drive currents of the SOA and the laser diode could be varied, since the coupler-combiner has a fixed value. In Fig. 5, it is shown that the decision point can be moved by adjusting only the drive current of the laser. A shift of about $4 \mathrm{~dB}$ with $2-\mathrm{mA}$ change in current was obtained.

Since we did the experiments with a discrete setup, the distance between the laser diode and the SOA was rather big, giving rise to a high round-trip time. This relative high round-trip time makes it difficult to perform dynamic measurements. It is planned to do some dynamic measurements on an integrated device in the near future.

\section{CONCLUSION}

We have proposed a new optical thresholding circuit that can easily be implemented. It consists of an SOA and DFB laser diode in a feedback scheme. Using this circuit a very steep decision characteristic was obtained both numerically and experimentally.

\section{REFERENCES}

[1] S. Verspurten, G. Morthier, and R. Baets, "Derivation of the amplitude histogram of an optical signal using an optical 2R regenerator," in ECOC-IOOC 2003, Italy, 2003, pp. 678-679.

[2] N. Calabretta, Y. Liu, F. M. Huijskens, M. T. Hill, H. de Waardt, G. D. Khoe, and H. J. S. Dorren, "Optical signal processing based on selfinduced polarization in a semiconductor optical amplifier," J. Lightw. Technol., vol. 22, no. 2, pp. 372-381, Feb. 2004.

[3] K. Nonaka, Y. Noguchi, H. Tsuda, and T. Kurokawa, "Digital signal regeneration with side-injection-light-controlled bistable laser diode as a wavelength converter," IEEE Photon. Technol. Lett., vol. 7, no. 1, pp. 29-31, Jan. 1995.

[4] M. Pantouvaki, M. J. Fice, R. Feced, E. P. Burr, R. Gwilliam, A. B. Krysa, J. S. Roberts, and A. J. Seeds, "10-Gb/s all-optical 2R regeneration using an MQW Fabry-Perot saturable absorber and a nonlinear fiber," IEEE Photon. Technol. Lett., vol. 16, no. 2, pp. 617-619, Feb. 2004.

[5] D. A. O. Davies, A. D. Ellis, and G. Sherlock, "Regenerative $20 \mathrm{Gbit} / \mathrm{s}$ wavelength conversion and demultiplexing using a semiconductor laser amplifier nonlinear loop mirror," Electron. Lett., vol. 31, pp. 1000-1001, Jun. 1995.

[6] D. Wolfson, P. B. Hansen, A. Kloch, T. Fjelde, C. Janz, A. Coquelin, I. Guillemot, F. Garorit, F. Poignt, and M. Renaud, "All-optical 2R regeneration at $40 \mathrm{Gbit} / \mathrm{s}$ in an SOA-based Mach-Zehnder interferometer," presented at the OFC-IOOC 1999, San Diego, CA, Feb. 1999, Paper PD36 suppl.

[7] J. De Merlier, G. Morthier, P. Van Daele, I. Moerman, and R. Baets, "Alloptical 2R-regeneration based on integrated asymmetric Mach-Zehnder interferometer incorporating MMI-SOA," Electron. Lett., vol. 38, pp. 238-239, Feb. 2002.

[8] O. Leclerc, B. Lavigne, E. Balmefrezol, P. Brindel, L. Pierre, D. Rouvillain, and F. Seguineau, "Optical regeneration at $40 \mathrm{~Gb} / \mathrm{s}$ and beyond," $J$. Lightw. Technol., vol. 21, no. 11, pp. 2779-2790, Nov. 2003.

[9] M. Zhao, G. Morthier, and R. Baets, "Demonstration of extinction ratio improvement from 2 to $9 \mathrm{~dB}$ and intensity noise reduction with the MZIGCSOA all-optical regenerator," IEEE Photon. Technol. Lett., vol. 14, no. 7, pp. 992-994, Jul. 2002. 